\title{
Comparative Study of the Cardiovascular Effects of Oral Hypoglycemics When Given Alone or in Combination: An In-Vitro Analysis
}

\author{
${ }^{1}$ Abdul Shabbir Ali Bhatti, ${ }^{2}$ Abdul Waheed Shehzad, ${ }^{3}$ Muhammad Ahmed Bhatti \\ ${ }^{I}$ Department of Pharmacology, Shalamar Medical and Dental College, Lahore \\ ${ }^{2}$ Department of Medicine, The Children Hospital and Institute of Child Health, Lahore \\ ${ }^{3}$ Department of Pharmacology, Avicenna Medical College, Lahore.
}

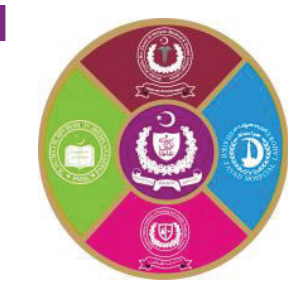

\begin{abstract}
Introduction: Cardiovascular disease is the major cause of mortality amongst the patients with T2DM (Type2 Diabetes mellitus). Any hypoglycemic agent can show serious adverse effects when given alone. eg; impaired myocardial function during MI (Myocardial infarction) with glimeperide and heart failure with pioglitazone. Metformin is cardioprotective and its combination with other OHAs (Oral hypoglycemic agents) is debatable. Aims \& Objectives: This experimental study was aimed to study the cardiovascular safety of OHAs when given alone and in combination. Place and duration of study: This study was conducted at Department of Pharmacology, Shalamar Medical and Dental College Lahore for a duration of 4 months (August - December 2017). Material \& Methods: The effects of graded doses $\left(10^{-9}-10^{-6} \mathrm{M}\right)$ of Metformin(M), Glimeperide(G), Vildagliptine $(\mathrm{V})$ and Pioglitazone $(\mathrm{P})$ were observed on retogradely perfused isolated rabbit hearts with warm Krebs-Henseliet solution on Radnoti working heart system. Forty two (42) rabbits were grouped into seven groups i.e; $\mathrm{I}(\mathrm{M}), \mathrm{II}(\mathrm{G}), \mathrm{III}(\mathrm{V}), \mathrm{IV}(\mathrm{P}), \mathrm{V}(\mathrm{M}+\mathrm{G}), \mathrm{VI}(\mathrm{M}+\mathrm{V})$ and $\mathrm{VII}(\mathrm{M}+\mathrm{P})$ each comprising of six animals(n=6). Effects of experimental drugs were observed on chronotropicity (HR), inotropicity (Peak rate of rise of LVP i.e; dp/dt) and Coronary flow (CF). The results were statistically analyzed with graph Padprism and wherever necessary paired or unpaired " $t$ " test was applied. Results: In groups $\mathrm{I}(\mathrm{M}), \mathrm{II}(\mathrm{G})$, and $\mathrm{IV}(\mathrm{P})$ significant $(\mathrm{p}<0.05)$ negative chronotropic effects were observed. However, glimepiride had stimulant effect on HR at higher concentrations. There was absolutely no change in Max $\mathrm{dp} / \mathrm{dt}$ in any group. In the first three groups of individual drugs and VI $(\mathrm{M}+\mathrm{V})$ there was significant decrease in coronary flow. Conclusion: Large doses of Glimepiride are potentially arrhythmogenic. Vildagliptine alone and in combination with metformin reduce coronary flow .These observations must be considered in patients of coronary disease.
\end{abstract}

Key words: T2DM, IHD, OHAs, Inotropicity, Max dp/dt

\section{INTRODUCTION}

$\mathrm{M}$ illions of people worldwide are suffering from diabetes mellitus. ${ }^{1,2}$ It was reported in 2011 by the International Diabetes Federation. This number is expected to rise in coming decades. ${ }^{3}$ Uncontrolled Diabetes mellitus is associated with increased risk of coronary and peripheral arterial disease. Tight glycemic control is associated with decreased mortility. ${ }^{4}$ Metformin significantly reduces lipid levels. Metformin is now considered safe in heart failure and renal failure as none of the patients allocated to metformin use in the UKPDS study (United Kingdom Prospective Diabetes Study) developed lactic acidosis. ${ }^{5}$ In clinical trials, safety of sulfonylureas has not been proved. Dipeptidyl peptidase-4 inhibitors have shown elevated level of cardiovascular risk. Rosiglitazone seems to have an increased risk of myocardial infarction and death. ${ }^{6}$ This experimental study was aimed to study the direct cardiovascular effects of OHAs when given alone and in combination.

\section{MATERIAL AND METHODS}

This experimental study was carried out in the laboratory of the Department of Pharmacology, Shalamar Medical and Dental College Lahore and conformed with the Guide for the Care and Use of Laboratory Animals published by the US National Institutes of Health (National Institutes Of Health Publication 85-23, revised 1996). 
Drugs used: Oral hypoglycemic drugs were procured from Wilshire pharmaceutical in the powder form free from any base.

Following treatments were employed:

Metformin (M), Glimeperide (G), Vildagliptin (V), Pioglitazone $(\mathrm{P})$, Metformin + Glimeperide $(\mathrm{M}+\mathrm{G})$,

Metformin + Vidagliptine $(\mathrm{M}+\mathrm{V})$, Metformin +

Pioglitazone $(\mathrm{M}+\mathrm{P})$

\section{Grouping of Animals}

42 normal male rabbits (weighing between $1.5-2 \mathrm{~kg}$ ) were purchased from the local market and divided into 6 groups. Group I (M), Group II (G), Group III (V), Group IV (P), Group V (M+G), Group VI $(\mathrm{M}+\mathrm{V})$, Group VII $(\mathrm{M}+\mathrm{P})$

\section{Preparation of Heart for Perfusion:}

Each rabbit was injected 5000IU (1ML) of heparin through the marginal ear vein one hour before slaughtering. Heart was removed from the chest after midsternal incision and immediately placed in an ice cold Krebs-Henseleit solution. The heart was mounted on Radnoti (Langendorff) working heart system via the aorta and its stump was secured for upto $3 \mathrm{~mm}$ on the grooved perfusion cannula by a silk ligature. A weight was attached to the heart apex and left to hang freely exerting resting tension of one gram. Heart was retrogradely perfused at a constant flow of $52 \mathrm{ml} / \mathrm{min}$ with warm KrebsHenseleit solution. It was allowed to stabilize for 30 mins in the perfusate which was equilibrated with 95\%O2 and 5\% CO2 with Krebs-Henseleit solution $\mathrm{pH}$ of 7.35 at $37^{\circ} \mathrm{C}$.

A saline-filled balloon catheter inserted into the left ventricle was used to measure isovolumetric work. Balloon was kept slightly larger than the maximum expanded volume of the ventricle to avoid measuring the resistance of the balloon to stretch. Each isolated heart was treated with four concentrations or doses $10^{-9} \mathrm{M}, 10^{-8} \mathrm{M}, 10^{-7} \mathrm{M}, 10^{-6}$ M.

\section{Statistical analysis:}

The data was analyzed by using Graph Pad Prism version 6.

\section{RESULTS}

1. There was decrease in heart rate with $\mathrm{G}\left(10^{-9} \mathrm{M}\right.$, $\left.10^{-8} \mathrm{M}, 10^{-7} \mathrm{M}\right)$ while effects produced with $\mathrm{G}\left(10^{-}\right.$ ${ }^{6} \mathrm{M}$ ) was significantly stimulant( Fig-1).

2. Combinations of Metformin + Vildagliptine decreased Coronary Flow (Fig-2) while Metformin + Glimeperide and Metformin + Pioglitazone had no effect.

3. Metformin, Glimeperide, Vildagliptine and Pioglitazone all decreased coronary flow (Table1)
4. There was no statistically significant difference in Max dp/dt between the experimental groups.

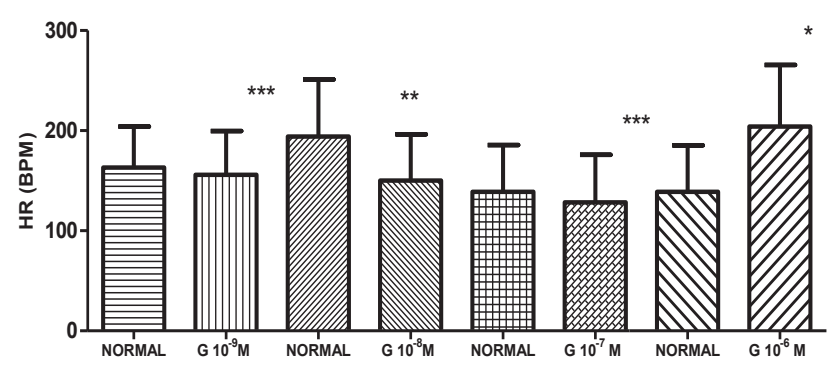

Fig-1: Effect of Glimeperide $(\mathrm{G})$ on heart rate

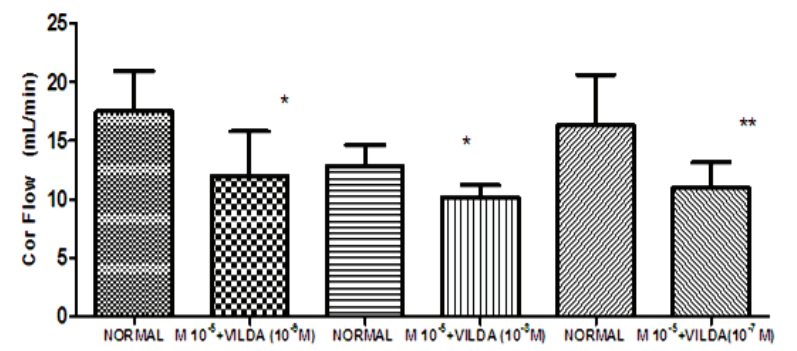

Fig-2: Effects of Metformin (M) + Vilda (V) on coronary flow

\section{DISCUSSION}

The effects of sulfonylurea on contractility, automaticity and adenylyl cyclase activity were studied on isolated rabbit atria by Pameret et al and Levey et al. ${ }^{7-10}$ The inotropic and chronotropic effects of sulfonylurea drugs are considered to be due to activation of adenylyl cyclase and hence increase in intracellular cyclic AMP. ${ }^{11-14}$ Glimeperide like other sulfonylureas has adverse cardiovascular effects. In our research, only chronotropicity was affected while there was no effect of individual drugs or combinations on inotropicity. Some oral hypoglycemics affect ischaemic myocardium. The dose-response effect of gliclazide and glibenclamide on ischaemia were investigated. The myocardium was adversely effected by glibenclamide at all concentrations and by gliclazide at supratherapeutic concentrations. These affects may have clinical implications in ischaemic heart disease.$^{15}$

Severe hypoglycemia as a result of intensive therapy for hyperglycemia may lead to increased mortality. Metformin reduces chances of myocardial ischaemia. ${ }^{14}$ Metformin is considered to be as firstline therapy in clinical practice guidelines. ${ }^{16}$ AMPK (AMP Kinase) is an important regulator of various cellular pathways and is considered to be a "master switch" for cellular energy levels. ${ }^{17}$ The cardioprotective actions of metformin are mediated through the activation of AMPK. 


\begin{tabular}{|c|c|c|c|c|c|c|c|c|c|}
\hline \multicolumn{10}{|c|}{$\begin{aligned} \text { Effects of Drugs } \\
\end{aligned}$} \\
\hline \multirow{2}{*}{\begin{tabular}{|c|} 
Drugs \\
METFORMIN(M) \\
\end{tabular}} & \multicolumn{3}{|c|}{ H.R (Beats Per Mins) } & \multicolumn{3}{|c|}{ Max dP/dt $(\mu \mathrm{V} / \mathrm{s})$} & \multicolumn{3}{|c|}{ Cor Flow(mL/min) } \\
\hline & Normal & Drugs & "p"Value & Normal & Drugs & "p"Value & Normal & Dugs & "p"Value \\
\hline $\mathrm{M}\left(10^{-9} \mathrm{M}\right)$ & 120 & 74.83 & 0.3051 & 33.16 & 33.16 & 0 & 24.67 & 9.233 & $0.0152 *$ \\
\hline $\mathrm{M}\left(10^{-8} \mathrm{M}\right)$ & 115.7 & 76.5 & 0.1594 & 33.16 & 33.15 & 0.3726 & 22.9 & 7.6 & $0.0162 *$ \\
\hline $\mathrm{M}\left(10^{-7} \mathrm{M}\right)$ & 125 & 34.83 & $0.0176^{*}$ & 33.16 & 33.16 & 0.3739 & 25.5 & 7.167 & $0.0002 * * *$ \\
\hline $\mathrm{M}\left(10^{-6} \mathrm{M}\right)$ & 152 & 74.17 & $0.0162 *$ & 33.15 & 33.16 & 0.3735 & 20.43 & 6.467 & $0.0018^{* *}$ \\
\hline \multicolumn{10}{|l|}{ VILDAGLIPTINE(V) } \\
\hline $\mathrm{V}\left(10^{-8} \mathrm{M}\right)$ & 139 & 127.3 & 0.5615 & 0.0174 & 0.0133 & 0.1803 & 19 & 5.8666 & $0.0094 * *$ \\
\hline $\mathrm{V}\left(10^{-7} \mathrm{M}\right)$ & 101 & 89.4 & 0.224 & 0.0176 & 0.0131 & 0.1242 & 20 & 5.56 & $0.0357 *$ \\
\hline $\mathrm{V}\left(10^{-6} \mathrm{M}\right)$ & 135 & 55 & 0.0639 & 0.0156 & 0.0119 & 0.5179 & 17.96 & 7.6666 & 0.0239 \\
\hline PIOGLITAZONE (P) & & & & 41.37 & & & 21.63 & & \\
\hline $\mathrm{P}\left(10^{-7} \mathrm{M}\right)$ & 107 & 113.8 & $0.0334 *$ & 41.37 & 41.37 & 0 & 21.67 & 7.633 & 0.0575 \\
\hline $\mathrm{P}\left(10^{-6} \mathrm{M}\right)$ & 74 & 52.33 & $0.0387 *$ & 41.37 & 41.37 & 0 & 22.9 & 7 & $0.0269 *$ \\
\hline $\mathrm{P}\left(10^{-5} \mathrm{M}\right)$ & 101 & 77.67 & 0.1648 & 49.64 & 41.37 & 0.3632 & 19.28 & 7.933 & $0.0204 *$ \\
\hline $\mathrm{P}\left(10^{-4} \mathrm{M}\right)$ & 72.8 & 63 & 0.659 & & 62.05 & 0 & & 4.95 & 0.0694 \\
\hline \multicolumn{10}{|c|}{ (Observations are means of six experiment) } \\
\hline
\end{tabular}

Beneficial myocardial metabolic effect of DPP-4 inhibition have been observed in mouse model of diabetes. Two weeks pre-treatment with either Vildagliptin or Sitagliptin manifested as reduced myocardial infarct (MI) size in animal hearts. ${ }^{18}$ A study with the DPP-4 inhibitor PFK275055, a vildagliptin-analogue, showed a reduced infarct size in pre-diabetic rats and sitagliptin showed increased infarct size. In one clinical trial involving patients with type-2 diabetes and cardiovascular disease, the addition of sitagliptin did not produce major adverse cardiovascular events or hospitalization for heart failure. ${ }^{19}$

In our study, it was observed vildagliptine like metformin, glimepiride and pioglitazone decreased coronary flow probably through vasoconstriction action. When vildagliptine was combined with metformin, effect was similar. However, combinations of metformin with glimepiride or pioglitazone did not show any significant untoward effects. The observed detrimental effect of the combination with sulphonylureas deserves further investigation. Our study produced variable experimental data as that obtained from other research. ${ }^{20}$ These effects are reflection of cardioprotective effects of metformin when given alone and in combinations except with vildagliptine. In one meta- analysis cardiovascular and heart safety profile of vildagliptine was explored ${ }^{21}$ in which no significant increased risk was found.

\section{CONCLUSION}

Combination of Metformin with glimeperide or pioglitazone tends to keep haemodynamic parameters within physiological limits. While Metformin and vildagliptine tend to produce ischaemic changes through coronary vasocostriction .It is advisable to use this safer combination therapy in all T2DM with IHD.

\section{REFERENCES}

1. Tahrani AA, Varughese GI, Scarpello JH, Hanna FW. Metformin, heart failure, and lactic acidosis: is metformin absolutely contraindicated? BMJ.2007, 335: 508-512.

2. U. K. Prospective Diabetes Study Group. Effect of intensive blood-glucose control with metformin on complications in overweight patients with type 2 diabetes (UKPDS 34). Lancet. 1998, 352:854-865.

3. UK Prospective Diabetes Study Group.Intensive blood-glucose control with sulphonylureas or insulin compared with conventional treatment and risk of complications in patients with type 2 diabetes (UKPDS 33). Lancet. 1998, 352: 837853.

4. Meinert CL, Knatterud GL, Prout TE: A study of the effects of hypoglycemic agents on vascular complications in patients with adult- 
onset diabetes. II. Mortality results. Diabetes. 1970, 19:789-830.

5. Nathan DM. Some answers, more controversy, from UKPDS. United Kingdom Prospective Diabetes Study. Lancet, 1998. 352: 832-833.

6. Nissen SE, Wolski K. Effect of Rosiglitazone on the Risk of Myocardial Infarction and Death from Cardiovascular Causes. N Engl J Med. 2007, 356(24): 2457-2471.

7. Palmer, R. F, K. C. Lasseter, and J. McCarthy. Tolbutamide: an inotropic effect on rabbit atria. Lanset. 1971, 1: 604.3.

8. Levey, G. S., R. F. Palmer, K. C. Lasseter. Effect of tolbutamide on adenylcyclase in rabbit and human heart and contractility of isolated rabbit atria. J. Clin. Enzdocrinol. Metab. 1971, 33: 371 .

9. Levey, G. S and S. E. Epstein. Activation of adenylcyclase by glucagon in cat and human heart. Circ. Res. 1969, 24: 151.

10. Levey, G. S and S. E. Epstein. Myocardial adenylcyclase: activation by thyroid hormones and evidence for two adenylcyclase systems. J. Clin. Invest. 1969, 48: 1663

11. Murad, F, Y. M. Chi, T. W. Rall, et al. Adenylcyclase. III. The effect of catecholamines and choline esters on the formation of adenosine 3',5' phosphate by preparations from cardiac muscle and liver. J. Biol. Chem. 1962, 237: 1233.

12. John W. Calvert, 1 , Susheel Gundewar, 1 SaurabhJha, et al. Acute Metformin Therapy Confers Cardioprotection Against Myocardial Infarction Via AMPK-eNOS- Mediated Signaling Diabetes. 2008, 57:696-705

13. Huisamen B, Genis A, and Marais E, A: Pretreatment with a DPP-4 inhibitor is infarct sparing in hearts from obese, pre-diabetic rats. Cardiovasc Drugs. Ther. 2011, 25 (1): 13-20. 10.1007/s 10557-010-6271-7.

14. Meinert CL, Knatterud GL, ProutTE. A study of the effects of hypoglycemic agents on vascular complications in patients with adult-onset diabetes. II. Mortality results. Diabetes. 1970,19:789-830.

15. Mahmoud Loubani, alan Fowler, Nicholas B, Standenb and Manual Galiñanesa .The effect of gliclazide and glibenclamide on preconditioning of the human myocardium European Journal of Pharmacology. 2005, 515(1-3):142-149

16. Hardie DG: Mini review: The AMP-activated protein kinase cascade: the key sensor of cellular energy status. Endocrinology. 2003, 144:5179-5183,

17. Hardie DG: The AM: P-activated protein kinase pathway-new players upstream and downstream. J Cell Sci. 2004, 117:5479-5487

18. Derek J Hausenloy, Hannah J Whittington, Abigail M Wynne, Shah S Begum, Louise Theodorou, Niels Riksen, Mihaela M Mocanu and Derek M Yellon. Dipeptidyl peptidase-4 inhibitors and GLP-1 reduce myocardial infarct size in a glucose-dependent manner. Cardiovascular Diabetology. 2013, 12:154

19. Jennifer B. Green., M. Angelyn Bethel., Paul W. Armstrong., John B. Buse., Samuel S. Engel., Jyotsna Garg., Robe. Effect of Sitagliptin on Cardiovascular Outcomes in Type 2. Diabetes. N Engl J Med. 2015, 373:232-242

20. Carolyn F, Daecon: A review of dipeptidyl peptidase-4 inhibitors. Hot topics from randomized controlled trials. Diabetes, Obesity and Metabolism. 2018, 20(S), 34-46.

21. Effect of metformin on cardiovascular events and mortality: a meta-analysis of randomized clinical trials. Lamanna C1, Monami M, Marchionni N, Mannucci. EEffect of metformin on cardiovascular events and mortality: a metaanalysis of randomized clinical trials. Diabetes Obes Metab. 2011, 13(3):221-8.

\section{The Authors:}

Prof. Abdul Shabbir Ali Bhatti

Department of Pharmacology,

Shalamar Medical and Dental College, Lahore.

Dr. Abdul Waheed Shehzad

Registrar, Department of Medicine

The Children Hospital and Institute of Child Health, Lahore.

Dr. Muhammad Ahmed Bhatti

$4^{\text {th }}$ year MBBS Students

Department of Pharmacology,

Avicenna Medical College, Lahore

\section{Corresponding Author:}

Prof. Abdul Shabbir Ali Bhatti

Department of Pharmacology,

Shalamar Medical and Dental College, Lahore.

E-mail: shabbiralibhatti@gmail.com 